Voix et Images

voixetimages

\title{
De Gilles Hénault à King Kong
}

\section{André Brochu}

Volume 11, numéro 1, automne 1985

\section{Naïm Kattan}

URI : https://id.erudit.org/iderudit/200546ar

DOI : https://doi.org/10.7202/200546ar

Aller au sommaire du numéro

\section{Éditeur(s)}

Université du Québec à Montréal

\section{ISSN}

0318-9201 (imprimé)

1705-933X (numérique)

Découvrir la revue

\section{Citer cet article}

Brochu, A. (1985). De Gilles Hénault à King Kong. Voix et Images, 11(1), 125-134.

https://doi.org/10.7202/200546ar d'utilisation que vous pouvez consulter en ligne.

https://apropos.erudit.org/fr/usagers/politique-dutilisation/ 


\title{
Poésie
}

\section{De Gilles Hénault à King Kong}

\author{
par André Brochu, Université de Montréal
}

Ce n'est pas un secret pour ceux qui affectionnent les potins littéraires du Devoir: j'ai pensé un moment transporter ma chronique dans une autre revue, n'étant pas sûr qu'elle fût à sa place dans ces pages. Voix et images se doit de rendre compte de l'actualité dans le domaine de la recherche critique avant tout. Le comité de rédaction, après une vigoureuse séance de réflexion, s'est entendu sur l'utilité de suivre aussi les actualités littéraires, mais elles seront couvertes de façon plus méthodique, le roman et le théâtre publié devant jouir désormais de la même attention que la poésie.

Autres remarques. Les lenteurs additionnées du service de presse, de la gestion éditoriale et de l'impression font que les recueils sont recensés au moins six mois et parfois un an seulement après leur parution. Cet inconvénient est cependant tout relatif. Un tel décalage permet en effet l'heureux rappel de recueils déjà soustraits aux feux rapides de la publicité. Notre but d'ailleurs n'est pas de créer la mode mais de présenter un tableau, trois fois l'an, de la production littéraire, tableau forcément partiel mais qui bénéficie déjà de quelque recul temporel.

Mes critères de sélection sont nombreux. J'accorde beaucoup d'importance, cela va de soi, à la qualité, de sorte que les éreintements sont rares. Mais il me faut aussi tenir compte de la représentativité, de la réputation des auteurs (ce qui ne veut pas dire la ménager) et chercher, à travers cela, à déceler et à encourager les jeunes talents prometteurs.

Quelques amis me trouvent bien doux. C'est eux qui sont féroces, et voudraient que le chef-d'œuvre régne rare sur un champ d'ossements. Pour ma part, tout langage vrai, où le travail et le don d'invention se fécondent mutuellement, m'émerveille et m'empêche de désespérer de ce Québec - ma patrie, mon dada - qui a besoin lui aussi d'indulgence, en ces temps postnationalistes et américanolâtres ${ }^{1}$.

Après vingt-trois ans de silence, Gilles Hénault, l'un des pionniers de la poésie moderne au Québec, publie ÂA l'inconnue nue ${ }^{\text {lts. }}$. Le rececueil est mince mais intéressant, sinon tout à fait séduisant. Les poèmes offrent une grande homogénéité d'inspiration. D'ailleurs ils reprennent tous le titre du recueil, qu'ils font suivre d'un numéro, de I à XXVI. Sept dessins de Bellefleur les illustrent magnifiquement.

Gilles Hénault n'a rien d'un lyrique ou d'un mystique même si, dans sa poésie, il se réfère explicitement à un intertexte symboliste et post-symboliste: Rimbaud, Mallarmé, Nelligan, Apollinaire. Quant à lui, il refuse tout merveilleux s'il n'est pas fondé sur la franche complicité de la matière. Le 
surréalisme et le marxisme ont été ses phares, dès cette époque où le Québec s'étiolait dans les liens d'un catholicisme unanime. Aujourd'hui, à soixantecinq ans, il chante la femme sans aucunement la mythifier, la femme charnelle et livrée, ou plutôt adressée au désir, mais non la femme-objet; plutôt celle qui dit à l'homme:

nous pouvons jouir autant que vous et peut-être plus... toute notre chair est un continent (quel mot) de jouissance. Nous pouvons aussi nous fermer à tout dialogue charnel... c'est nous qui décidons de votre entrée dans le monde, mon ami (hors le viol).

Cette citation montre que le poème peut accueillir un discours passablement abstrait, d'ordre sapientiel ou idéologique. En général, le propos est plus fluide, plus polysémique et fait un usage abondant de l'homophonie (paronomase, homonymie, écho, archilexie, calembour...). Le seul poème XXI contient les figures suivantes: orgasme de barbarie -le paysage gire et gicle - remous et remuements argentés des poissons - l'amer amour ne t'a donc pas noyée dans les frayères dans les frayeurs du corps profond - car la nue dans la rade signale l'Inconnue nue - la vague divague. Le procédé irriterait s'il ne suggérait la parfaite clôture du réel: les mots les plus différents quant au sens, grâce à leur matérialité cessent de se fuir, avouent leur fondamentale identité. Ou plutôt, puisque l'identité est un leurre métaphysique, ils explicitent simplement le jeu de leur différence, fondé sur des grammes, (gramma, lettre) c'est-à-dire du mesurable. Le mystère est évincé - du langage, de l'amour, de l'existence. Le poème est une plage où le discours s'invente librement, sans trop de folie ni de beauté. Nu. Comme essentiel.

Dans la collection "Rétrospectives" l'Hexagone accueille un nouveau titre: Souvenirs Shop, de Jacques Godbout ${ }^{2}$ qui, depuis un quart de siècle, est passé pour de bon à la prose, sans velléité de retour à ses premières amours. Aussi la dernière section du recueil, datée de 1980 , atteste-t-elle l'orientation de l'écrivain, tout en retrouvant quelque chose de l'inspiration poétique. Ces «proses» évoquent en effet de menus souvenirs, par exemple le parc Belmont, les premières initiales gravées au couteau sur un pupitre, des images de l'Ethiopie... Représentations diverses, fugaces (Godbout touche à tout) que l'auteur appellerait volontiers des textelets, et qui rappellent les poèmes aux airs de chansonnettes de Carton-pâte ou des Pavés secs. Un thème pourtant en fait l'unité: celui du coin de pays, cherché partout, jusqu'en Afrique et trouvé finalement dans ma langue à nous c'est-à-dire dans l'écriture, seule capable de fonder l'appartenance et de mettre en rapport une patrie et l'univers.

Mais qu'en est-il des poèmes, les vrais, par lesquels Godbout a fait son entrée en littérature? On s'attendrait à ce qu'ils aiènt mal vieilli, leur auteur donnant toujours l'impression d'établir ses quartiers dans le provisoire. Or il 
faut constater, au contraire, que la fraîcheur des textes subsiste, s'alimentant à des profondeurs insoupçonnées. Rien de trop dans ces brèves esquisses mentales ou narratives, d'une grande netteté d'exécution. Sans doute n'y sentons-nous guère le grand souffle lyrique, encore moins la tension épique. Godbout cultive le sujet petit, l'idée charmante et ingénieuse, l'énigme qui fait rêver, le mot qui dérange, provoque, émeut. Il dit les bonheurs vifs et les mélancolies pudiques, souvent la haine de la mort. De travailler ainsi en miniature - C'est la chaude loi des hommes échappe cependant à une telle observation, avec ses poèmes plus longs et, dirait-on, menacés d'éclatement - permet au poète la maîtrise absolue de son texte. Godbout, en effet, cherche généralement à rester supérieur à ce qu'il écrit, quitte à en limiter l'envergure. Et ses textes sont comme hantés par la grandeur qu'ils refusent. Peut-être est-ce elle, justement, qui les gorge en secret et les rend aptes à défier le temps.

La modernité s'est longtemps complu à interroger l'écriture comme langue, comme usage des signes. C'était la phase abstraite, formaliste, qui permit la découverte d'un continent nouveau, celui des mots, aussi vaste que celui des choses. Mais l'attention au code requérait qu'on tournât le dos au réel et qu'on offusquât la lisibilité, pour mieux faire apparaître les nervures du langage. Aujourd'hui, les recherches de pointe entendent réinvestir les mots dans le réel, et l'accent se déplace significativement du code vers le support matériel qui assure l'inscription de la communication dans le vécu. Dans les Rendez-vous par correspondance, suivi des Prénoms, Louise Cotnoir ${ }^{3}$ imagine un genre astucieux, le poème par lettres (formule qu'elle utilise cependant de façon surtout allusive); elle y tire tout le parti possible de la situation épistolaire pour explorer la relation à l'autre - et à soi - telle que le message écrit la réalise.

La deuxième partie du livre prolonge la première en ce qu'elle donne rendez-vous, par la magie des prénoms, à toute une théorie de figures aimables et aimées, femmes proches et lointaines qui peuplent l'imaginaire et la pensée de l'écrivain à la façon de mots-choses merveilleux, inépuisables:

tous les siècles, toutes les langues

affectionnent mes prénoms

en français, en hébreu, en anglais

je possède une perception primitive

qui garde les jeux intacts de l'enfance.

Il y a, dans la poésie de Louise Cotnoir, une chaleur, une exigence de vie intègre et libre qui tirent certainement leur origine de l'enfance, plus précisément d'une complicité avec la mère. C'est elle, la mère, qui fonde la lignée de femmes braves, / audacieuses, originales, (qui) m'enseigne/l'indépendance, l'intégrité et le respect. Et pour ceux qu'effraierait ce langage moral, ou cette référence à ceux qu'on aime et qu'on gratifie de présence réelle dans l'Histoire, rappelons que Louise Cotnoir renoue ici avec une 
tradition poétique fort valable, qu'a illustrée par exemple Victor Hugo dans ses poèmes intimistes des Feuilles d'automne. L'être humain a toujours rêvé que les noms et prénoms de son amour aient l'universelle résonance des noms communs: astre, ruisseau, murmure...

Nous ne serons jamais intacts, de Guy Moineau ${ }^{4}$, est un mince recueil de proses séduisantes, énigmatiques, où quelques situations du vécu personnel ou amoureux sont fugacement esquissées. Par exemple:

Vie normale. Je balbutie les unités volontaires de la mort.

(...) Regard exténué sans tes verres, la signification des dommages te tourmente. Indéfiniment.

Lors de la photographie officielle, nous adoptons le touch me if you can attitude.

On sent là beaucoup de détachement, comme si la vie était tenue à bout de bras, et prétexte à des phrases parfaites mais inutiles. Pourtant il y a là de la beauté, de l'inédit. Je balbutie les unités volontaires de la mort est un énoncé plein de résonances, où s'équilibrent magiquement le concret et l'abstrait, où se fondent le lisible et l'illisible.

La poétique de Moineau est essentiellement déceptive, comme celle de Roger des Roches ou de Normand de Bellefeuille. Elle génère des formules éclatantes, qu'on enrage de ne pouvoir rapporter à autre chose que du langage, que le Langage, Centre du texte. Centre-et-ailleurs. Nulle part.

Des formules, Jean Charlebois a le don lui aussi mais elles ne sont jamais l'effet ou l'occasion d'une mise à distance du vécu. Au contraire: le monde est là, à prendre à pleines mains. Le corps est tout entier l'organe de la perception, car il est le lieu d'un jouir franc et constant. La pornographic, dont on lit la voix en contre-chant du poème, a l'accent de la vraie pureté: sans mélange, le désir assure la présence à tout. Présent $!^{5}$ est le titre de ce recueil rouge flamme, dont les pages couvertures sont vierges d'inscriptions - nues comme des mains.

Sur les pages de gauche, on lit des communiqués de presse, des citations, des notations schématiques, généralement datées, comme s'il s'agissait d'un journal; mais un journal extime, où prime la réalité objective. C'est, si l'on veut, une évocation du monde tel qu'il s'offre, jour après jour, à la conscience de chacun.

Les pages de droite comportent, en haut, une bribe de discours amoureux, très cru, puis le poème proprement dit, ainsi non dissocié du nonpoème qu'est le texte du réel (page de gauche) et de la voix du désir qui appartient à une autre scène, ob-scène, pulsionnelle et, pour ainsi dire, matérielle. Le dispositif textuel a donc pour effet de relativiser le poème en le 
rapportant à ses autres, mais ce faisant, il fait mieux ressortir le caractère miraculeux (et simple) d'une parole à la fois gratuite et nécessaire, dont les mots ont le poids de la chair;

Embaume, amour, en cet air aboli.

Brame, amour.

Jaillis des hanches.

(...) Abonde, amour:

substitue-toi à l'air.

Fais l'espace et l'eau.

Depuis toujours Cécile Cloutier pratique, en poésie, l'art périlleux de la miniature. Voici l'Échangeur ${ }^{6}$, au titre symbolique: les échangeurs sont les carrefours d'autrefois, adaptés à nos temps de vitesse folle et de jonctions vertigineuses. Soixante-dix poèmes, avares de mots et d'idées, proposent de petites scènes de genre dont les actants sont des abeilles (L'épice/D'une abeille/Sur la peau du ciel / Éveille / La saveur du cri), les pivoines (L'accalmie / De la soie / Enveloppait le monde / / Les pivoines rêvaient), etc. Univers peuplé d'objets souvent aigus, prompts à se muer en écriture comme dans le beau, le saugrenu poème qui suit:

Piquent les fougères du songe

Sur les dimanches de satin gris

Un encrier commence

On peut rêver longtemps, dans les mailles du texte. Son laconisme serait ennuyeux s'il dissimulait un Message, une métaphysique. Nenni: juste ce qu'il faut de symbole pour lester la nacelle de la suggestion, empêcher le voyage sans retour vers les étoiles.

Que nous disent, en fin de compte, les petits rébus de Cécile Cloutier? Eh bien! elle, justement; ses hantises de cuivre, de soie, de satin, d'abeilles, de fourrure. Et une grâce, sur tout cela: celle d'écrire juste au niveau où écrire se peut, sans un mot de trop - il serait épouvantable! - mais avec tous ceux qu'il faut, propres, nets, comme des politesses à faire au silence, à la mort.

Entre l'inerte et les clameurs, Gilbert Langevin ${ }^{7}$ poursuit sa quête de ce qui compte et qui s'appelle, simplement, amour. Le mot apparait au terme d'un recueil où s'équilibrent l'expression du découragement, face à un présent calamiteux, et celle, malgré tout, de l'espoir, encore capable d'inspirer la révolte. C'est cela, l'inerte et les clameurs: le tic et le tac d'une existence qui doit trouver en elle-même ses raisons de durer, car le paysage social n'a rien de reluisant. On vit dans la vacuité dans l'inconfortable / aux perspectives désaffectées, et il n'est peut-être pas sans pertinence de traduire ce 
constat en termes politiques (après-référendum, virage fédéraliste du parti québécois, érosion des aspirations collectives, etc.). Suit, dans le même poème, une notation d'une grande justesse concernant la réaction individuelle, celle du créateur, à la conjoncture globale: l'imaginaire en colère / étrangle toute musique interne. Avant de commander le sursaut libérateur, l'imaginaire se retourne d'abord contre les objets immédiats de la psyché: de toute façon, rien, à l'extérieur, ne lui offre une résistance. Il lui faudra de nouveau ensemencer patiemment le réel pour y réimplanter les chances d'une action.

En attendant, la description attentive du présent, si délabré soit-il, permet déjà, par le travail du langage, la mobilisation des sentiments humains. On ne peut rester insensible devant une expression de la détresse aussi sobre et poignante que celle-ci:

Il y a voie noire

il y a tache de sang dans l'oeil de la tempête

plein fouet sur des corps malades

terre à pitié

paysage de fête morte

il y a tristesse ardoise et la sérénité

se cache derrière un miroir fatidique

N'y voyons pas le retour au noir métaphysique dont Saint-Denys Garneau a barbouillé notre poésie, du reste avec génie, en un temps fort propice aux lamentos. Chez Langevin, la sérénité subsiste, même occultée. Elle s'alimente à l'intime feu des rêves / étincelle natale, où prennent racine toutes puissances d'aimer.

On prend la mesure du classicisme de Langevin quand on se trouve ensuite confronté au baroquisme effréné de Lucien Francœur. Son dernier recueil, Exit pour NomadeS (sic) ${ }^{8}$, reprend les thèmes des Rockers sanctifiés ${ }^{9}$, de façon plus articulée cependant. Les innombrables hors-d'œuvre, citations, photographies, ont disparu et le texte a heureusement reconquis ses droits. Pas toujours bien léché, certes; violemment hétéroclite: J'écris n'importe quoi n'importe où n'importe comment. On croirait que Francœur tient simultanément les discours de Chamberland (le mutant), de Denis Vanier (le tatouage), de Yolande Villemaire (l'Égypte), de Claude Beausoleil (la ville) et de quelques autres, le tout épicé de références fort intellectuelles à Deleuze et Guattari, Pélieu, Saint-John Perse, Teilhard de Chardin... Rocker, Francoeur adopte le style motard en poésie, fait grand tapage autour de quelques idées pauvres, par exemple que Dieu et le sexe sont une même chose; mais Francoeur, derrière son personnage, est un jeune homme rusé, intelligent, capable de grands raffinements de pensée et de langage. Il cultive son personnage parce qu'il lui permet de rêver, de prolonger sciemment son adolescence, de cueillir les fruits blets du narcissisme, de revendiquer une androgynie (ici on n'est pas loin de Jacques Renaud et de son merveilleux Clandestine(s) ${ }^{10}$ ) dont on ignore si elle constitue un élargissement de la 
sexualité ou une franche régression. Mais même si Francoeur semble souvent verser dans l'infantilisme, son inspiration témoigne d'un courage certain. Bien entendu, ii est loin de la délinquance d'un Vanier, aristocratique mais réelle. Mais on ne trouve que chez lui ces motifs qui composent la petite misère quotidienne d'une jeunesse sans argent et sans travail, inapte à vivre - ce qu'il appelle, par exemple, l'automagnification manuelle et qui est, tout simplement, la masturbation. Tout cela coiffé d'une tranquille, souriante proclamation d'égoïsme:

Une obsession bien dirigée

Commence toujours

Par soi-même

Qui d'autre que Francoeur oserait, aussi facilement, se réclamer de l'Ouroboros, ce serpent des légendes, aux connotations fort lubriques, qui se dévorait lui-même?

Être rocker, c'est être le partisan exigeant des facilités, se laisser aller aux bruits et aux fureurs de la jeunesse, de la ville, de l'Amérique. Passé un certain âge, il y faut de l'acharnement, et on ne peut dénier, à la poésie de Francoeur, une réelle vitalité. C'est là sa principale beauté. Elle n'est nullement à dédaigner.

Non moins acharné, Paul Chamberland poursuit le grand projet de refaire le monde et l'homme en détournant à cette fin les inventions et découvertes scientifiques, les pratiques sociales transgressives, tout ce qui peut combattre la "dégradation de la vie" (titre d'une des deux parties du livre). Compagnons chercheurs ${ }^{11}$ appartient donc, comme les grands livres de Chamberland - Demain les dieux naittront, le Prince de Sexamour, Extrême survivance, extrême poésie, lesquels ne sont pas, à mon avis, ses œuvres les plus réussies au plan littéraire - à la veine prophétique. 11 me semble cependant que Compagnons chercheurs, tout en restant fidèle à l'espoir démesuré d'une mutation de l'homme, de l'avènement d'un tout-autre, retrouve le sol des raisons culturelles, que j'oppose aux fabriboles contreculturelles dont le défroqué de Parti pris s'est longtemps repu. Chamberland me semble renouer ici avec une part de son passé, non celle du politique mais celle, antérieure, du philosophe, soucieux d'explorer le rapport de l'homme au monde et d'ouvrir des avenues nouvelles à la pensée et à l'action - sur la base d'une juste perception du réel. La poésie peut être alors poésie morale, réflexion sur les signes du temps, dénonciation de l'Engrenage zombernétique et de l'universel Désagrégat, appel à la formation d'une communauté, un NOUS, dont le champ constitutif est l'espèce humaine. La poésie est dès lors pleinement poïèsis, c'est-à-dire action, Verbe incarné - un Verbe tout humain.

Le réalisme reste cependant timide et Chamberland ne dédaigne pas de recourir, une fois de plus, au mirage du sectarisme et d'imaginer on ne sait quel égrégore comploteur, noyau de vigilants qui s’étend en réseau expansif et agile à travers tout le tissu sociorganique. 
La deuxième partie, "L'humiliation de l'amour», parmi d'abondantes citations, contient quelques belles pages qui disent l'amour en mots de chair et de feu, doux ébranlement de l'intime à l'intime.

Je suis cet homme qui debout au milieu de ses phrases ne sait que dire, s'alimente de silence, cet homme étrange au milieu duquel la surréalité abaisse ses antennes pour mieux VOIR. Cette phrase est tirée de Parler ne s'entend pas, de Denuis Saint-Ýves ${ }^{12}$. L'originalité de certains discours, qui rappelle celle de certains prénoms..., fait qu'on hésite, incapable de décider si l'on doit la rapporter à une exigence profonde ou à un système artificiel de fabrication du texte, comme il y en a tant de nos jours. Je n'avais pu me faire une idée assez nette sur le recueil pour en risquer un commentaire. Devant Orifices ${ }^{13}$, mes doutes ne sont pas tous levés et j'ignore si la solitude du poète justifie vraiment l'étrangeté de son discours, ou si ce n'est pas plutôt le parti pris de déconcerter qui condamne la parole à l'incommunicabilité. Je prends plaisir, cependant, à ces menus propos aux airs de confidence, quelques-uns dédiés à Léo Ferré; où la ponctuation, franchement impertinente, crée dans le suivi de la prose une discontinuité qui pourrait bien équivaloir à la découpe du vers libre:

j’ouvre la fenêtre. Gaspé, ressemble, à une étoile, de mer. toute émotion toute rotation. je me laisse porter par l'air frais qui vient de là-bas. j'enfile, un vieux chandail.

On imagine, plutôt que les virgules, un renvoi à la ligne:

jouvre la fenêtre. Gaspé

ressemble

à une étoile

de mer (etc.)

La virgule dit la pulsion, non le sens, elle prolonge le je désirant dans les mailles du texte, comme un fil de trame rendu visible. Le texte, autour, peut devenir carrément hermétique:

toute trouée, vers des hypothèses de nerf(s), me surprend, m'enivre, me seconde, et toi? je ne toise jamais seul. tout dit la révérence à moins que. mais passons. j'appréhende un dire intrinsèque.

Ce dire intrinsèque est sans doute le chant privé du désir, que la poésie tente de signifier au plus près. Ce faisant, elle trahit peut-être sa mission, qui est de rendre le désir humain, c'est-à-dire désirable, non de le reproduire tel quel. La poésie ne copie pas, elle crée le vécu.

La dernière œuvre de François Charron, François ${ }^{14}$, se compose de trois textes d'inégale longueur. Celui du milieu, le pius long, donne son titre au livre. Le tout relève du poème narratif en prose, et non du récit poétique car 
la dimension proairrétique (ou code des actions, dans le jargon de Barthes) y est nettement subordonnée à une intention poétique, non lyrique certes mais divagante, au sens mallarméen. C'est une divagation en effet, c'est-à-dire un genre de fantaisie où la représentation est trouée, déconstruite à mesure, contaminée d'intentions intellectuelles, qui porte ce flot d'écriture au long de cent pages quelque peu ennuyeuses, il faut bien le dire. Quand on a la facilité, ou mieux l'aisance de Charron, un peu de génie est de mise sinon l'écriture devient une pâle copie d'elle-même. Ce qui me semble manquer cette fois, c'est cela qui troue la page, qui fait noeud dans le sens, qui dérange et déporte, fait rugir les circuits. On n'a droit qu'à l'évocation sibylline du vécu d'un jeune garçon. François, qui est et n'est pas le narrateur; il aime Sophie qui change de nom et s'appelle désormais Monique; il y a aussi des comparses: le personnage, le frère et la sœur (de Madeleine), le guerrier, la poupée, ombres fugitives dans une histoire inconsistante, sans grand intérêt, pur prétexte à écrire. Écrire pour écrire.

Encore faut-il trouver des lecteurs qui se satisfont de lire pour lire, de s'égarer sans profit dans la rhétorique impénitente d'un producteur de langage. Certes, Charron n'est pas que cela et il a fait maintes fois déjà la preuve de son talent et de son sérieux. Dans l'ensemble, malgré la complaisance qui l'entache, son œuvre est une des plus stimulantes qui soient, et des plus dignes d'être aimées. Mais François pousse si loin le bonheur égoïste d'écrire qu'il débouche en pleine insignifiance. On lit alors des billevesées de ce genre: Mon air malheureux descend dans la piscine et l'eau s'agite. Je recouvre la santé. Je me sèche, me peigne et me change. Voilà une belle idée. On est loin de ce régal métaphysique que promet le prière d'insérer.

Michel Muir vient de rendre un service inestimable aux Herbes rouges en publiant, contre cette dynamique maison d'édition à qui nous sommes redevables de plusieurs titres qui font date, une charge d'une bêtise phénoménale. Cela s'intitule Poètes ou imposteurs?'15 et semble issu de plumes d'un autre âge, celles d'un Roger Brien ou d'un Jules-Paul Tardivel, voire de Gilberte Côté-Mercier dans ses moments d'illumination les plus décidés. Un spiritualisme d'inspiration pathologique éclate à chaque page. Je ne perds pas mon temps à citer. Je déplore simplement l'occasion perdue. Les Herbes rouges méritaient une remise en question sérieuse, propre à relancer le débat sur la nature et sur les fins de la poésie, fût-ce au prix de quelque polémique. Mais entre la polémique et l'ouvrage de Michel Muir, il y a la distance qui sépare Henri Meschonnic de King Kong. 
1. Cette position est résumée correctement par Jean Basile, dans l'article par ailleurs ignoble, le mot n'est pas trop fort, qu'il a consacré à mon Langevin ( $c f$. La Presse, 22 Juin 1985, «Plus», p. 16).

1. bis Gilles Hénault, Ȧ l'inconnue nue, Montréal, Parti pris, 1984, n. p.

2. Jacques Godbout, Souvenirs Shop, poèmes et proses 1956-1980, Montréal, l'Hexagone, 1984, $210 \mathrm{p}$.

3. Louise Cotnoir, les Rendez-vous par correspondance suivi des Prénoms, Montréal, les Éditions du Remue-ménage, 1984, 100 p.

4. Guy Moineau, Nous ne serons jamais intacts, Montréal, revue les Herbes rouges, No $133,1985,28 \mathrm{p}$.

5. Jean Charlebois, Présent! Saint-Lambert, le Noroît, 1984, 108 p.

6. Cécile Cloutier, L'Échangeur, Trois-Rivières, Écrits des Forges, 1985, $80 \mathrm{p}$.

7. Gilbert Langevin, Entre l'inerte et les clameurs, Trois-Rivières, Ecrits des Forges, $1985,52 \mathrm{p}$.

8. Lucien Francoeur, Exit pour NomadeS, Trois-Rivières, Écrits des Forges, 1985, 108 p.

9. Lucien Francoeur, les Rockers sanctifiés, Montréal, l'Hexagone, 1982, 350 p.

10. Jacques Renaud, Clandestine(s) ou la Tradition du couchant, roman, Montréal, Le Biocreux. 1980. 504 p.

11. Paul Chamberland, Compagnons chercheurs, Longueuil, le Préambule, 1984, 130 p.

12. Denuis Saint-Yves, Parler ne s'entend pas, Trois-Rivières, Ecrits des Forges, 1981, n.p.

13. Denuis Saint-Yves, Orifices, Trois-Rivie)res, Écrits des Forges, 1984, 70 p.

14. François Charron. François, Montréal, les Herbes rouges, 1984, 108 p.

15. Michel Muir, Poètes ou imposteurs? Montréal, Louise Courteau éditrice, 1985, 176 p.

P. S. Michel Beaulieu est mort.

Il y avait en lui un homme avantageux. Cet homme cachait un être tendre et très simple, l'enfant qu'il n'avait cessé d'être.

Le voici, dans nos aubes grises. Ses livres restent, qui nous parlent de lui, de nous. 